\title{
Phenytoin induced Stevens Johnson Syndrome: A Case Report
}

\author{
Upinder Kaur ${ }^{1}$, Manish Bansal ${ }^{2}$, Ajit Kumar ${ }^{3}$, Sankha Shubhra Chakrabarti ${ }^{4}$, Dinesh Kumar and BI Pandey $^{6^{*}}$ \\ 'Department of Pharmacology, Institute of Medical Sciences, Banaras Hindu University, Varanasi-221005, UP, India.
}

\begin{abstract}
Stevens Johnson syndrome (SJS) is a disastrous consequence of hypersensitivity reaction precipitated by certain drugs and viral infections. Among the drugs, antiepileptics, non-steroidal anti-inflammatory compounds and antibiotics are commonly implicated in the pathogenesis of SJS, although the overall incidence of this entity is very low. The syndrome is characterizedby purpuricmaculesand bullous eruptions involving the mucous membrane which may be followed by systemic manifestations. The disease may be lethal if undiagnosed and untreated. Age of the patient, area of involvement, nutrition status and presence of co-morbidites are the important determining factors of prognosis. Septicemia and gastro intestinal bleeding are the lethal complications seen to develop if the disease remains untreated. Though not universal, but HLAB*1502 allele has been linked with phenytoin induced SJS in certain populations. Despite years of research, mechanism of SJS is not fully defined. Immune dysfunction mediated by Tlymphocytes in response to phenytoin and oxi-
\end{abstract}

dative stress generated by phenytoin are thought to be responsible. Here we present a case of SJSinduced by phenytoin in an adult female. The case warrants the need of adopting a meticulous approach while prescribing phenytoin, especially in females who are at increased risk of developing drug relateddermatological adverse effects.

Key words: Stevens Johnson syndrome, Phenytoin, Anti-epileptics, Pharmacovigilance.

\section{Address for Correspondence:}

Professor BL Pandey, Department of Pharmacology, Institute of Medical Sciences, Banaras Hindu University, Varanasi-221005, UP, India.

Phone no: 9451440039

E-mail: blp53@rediffmail.com

DOI : 10.5530/PTB.2016.1.6

\section{INTRODUCTION}

Stevens Johnson syndrome(SJS) and toxic epidermal necrolysis(TEN) are rare but lethal manifestations of hypersensitivity reaction which develop in response to infections like herpes or more commonly in response to drugs. ${ }^{1}$ The full blown form of the syndrome is preceded by a prodromal phaseof flu like symptomswhich evolve over 1-3 weeks to cutaneous manifestations in the form of macular eruptions over the trunk, face and upper limb. ${ }^{2}$ Involvement of mucous membrane is seen in $90 \%$ of cases. ${ }^{3}$ Diagnosis is made on clinical suspicion and confirmed by biopsy. Prognosis depends upon the age of patient, presence of comorbities and area of detachment. ${ }^{2}$ Septicemia is the most common complication of SJS and the major contributor towards mortality. ${ }^{3}$

SJS and TEN are two variants of the same condition differing only in the percentage of skin detachment. Usually $<10 \%$ body surface area (BSA) involvement is seen in SJS, 10-30\% BSA in SJS-TEN overlap and $>30 \%$ BSA detachment is seen in TEN. ${ }^{3}$ Among the drugs, anti-epileptics, antibiotics like sulfonamides and isoniazid, NSAIDS are the common culprits. ${ }^{1}$ Here we report a case of SJS in a 30 year female after she was put on prophylactic dose of phenytoin.

\section{CASE REPORT}

A 30 year old lady was put on prophylactic phenytoin by neurologist after she met with head trauma. Phenytoin was given at the dose of 100 mg orally thrice daily.After about 10 days of phenytoin therapy, the lady started developing oral and lip erosions with crusting. This was followed in 2-3 days by macularlesions over the face, bilateral upper limbs and upper trunk.She was then shifted to the department of dermatology. There was no history of fever, irritant application, bullae formation or self-medication. On examination, thepatient was afebrile with body temperature of $98.2^{\circ} \mathrm{F}$, pulse- $90 / \mathrm{min}$, respiratory rate- $18 / \mathrm{min}, \mathrm{BP}-120 / 70$ $\mathrm{mm} \mathrm{Hg}$. Maculopapular hemorrhagic lesions in healing phase were seen on face, bilateral upper limbs and upper trunk with BSA involvement of $7-8 \%$. (Figure1, 2)Haemorrhagic lip crusting and oral ulcers were present along with whitish removable deposits on tongue. She also had periocular edema and conjunctival redness. Photographs (Figure 1,2) were taken after obtaining informed consent from the patient.Investigations done on D1 and D3 are shown in Table 1. Diagnosis of SJS with oral candidiasis was made on clinical basis. SCORTEN score was done on day 1 and day 3 and was 0 on both the days. ${ }^{4}$ Patient was managed using intravenous fluids, antifungals and antibiotics with careful monitoring of vitals and routine biochemical parameters. Clobazam was given at the dose of $10 \mathrm{mg} /$ day. Patient was given topical steroid, antibiotic and normal saline compresses for local application over the lesions. In addition, xylocaine gel and lubricant-antibiotic eye drops were given for oral ulcers and conjunctival inflammation respectively. On discharge, patient's condition was significantly improved.

\section{DISCUSSION}

SJS and TEN are rare but lethal manifestations of a type IV hypersensitivity reaction with an approximate incidence of 1-2/million/year. ${ }^{1}$ SJS is associated with a mortality rate of $1-5 \%$ which increases to $25-35 \%$ in case of TEN. ${ }^{1}$ Risk of developing SJS after anticonvulsants is maximum in the first 2 months of therapy. ${ }^{5}$ Among antiepileptics, those with aromatic structure and long half lives are commonly involved in the precipitation of SIS.In addition there is a high degree of cross reactivity among the anti epileptics. ${ }^{5}$ A study has shown that the risk of developing cutaneous adverse drug reaction(CADR) is high in females ${ }^{6}$ In addition phenytoin and carbamazepine have been linked with higher severe to non-severe cutaneous reaction ratio. ${ }^{6}$ Here, we presented a case of SJS in a 30 year female who was taking phenytoin. Another condition that can be confused with drug induced SJS is drug induced pemphigus foliaceous. The onset of lesions within 15 days of taking phenytoin and the maculopapularnature of lesions with oral involvement seen in this patient make pemphigus foliaceousunlikely.The latter entity often bears association with thiol group of drugs. ${ }^{7}$

Mechanism of SJS is uncertain but has been linked to immune dysfunction. It is presumed that owing to some genetic defect, there is altered metabolism of drug and its interaction with the immune components which provokes the catastrophic reaction.CD8+ cytotoxic T Lymphocytes are 


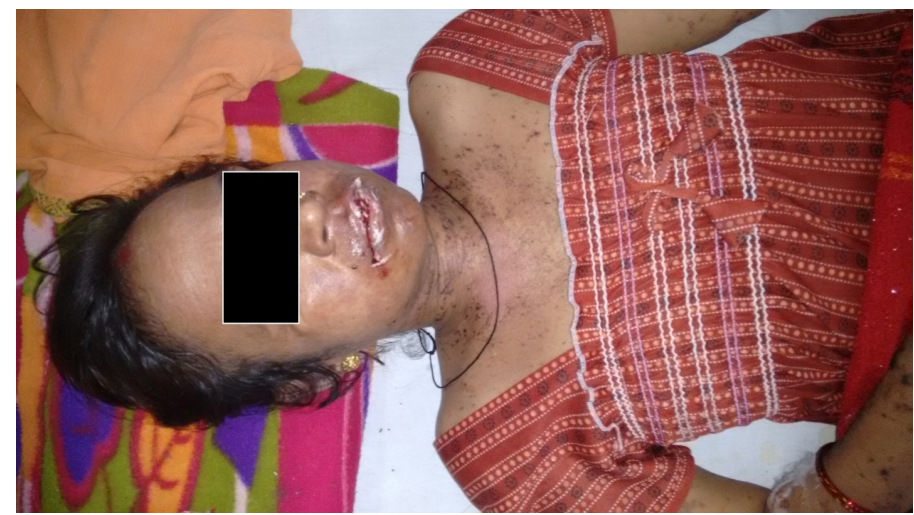

Figure 1: Oral erosions and haemorrhagic lesions in healing phase over upper trunk and upper extremities

Table1: Investigations of patient on day 1 and day3 of hospital stay

\begin{tabular}{ccc}
\hline Investigations & Day 1 & Day3 \\
\hline Hb $(\mathbf{g m} / \mathbf{d l})$ & 10.8 & 10.1 \\
TLC $\mathbf{P C}(/ \mathbf{\mu l})$ & 5900, 3.27 lakh & 5500,3.6lakh \\
DLC & N46L19M29E6 & N67L30M2E1 \\
SGOT/SGPT(mg/dl) & $103 / 81$ & $96 / 103$ \\
RBS(mg/dl) & 89 & 98 \\
ALP,TB/DB(mg/dl) & $328,0.7 / 0.2$ & $345,0.8 / 0.3$ \\
TP/ALB(g/dl) & $5.2 / 3.1$ & $4.5 / 2.7$ \\
Urea/creatinine & $10.1 / 0.65$ & $5.6 / 0.7$ \\
Na/K/Cl(meq/l) & $133 / 4 / 98.6$ & $139 / 4.3 / 104$ \\
Serum HCO $\mathbf{3}(\mathbf{m m o l}), \mathbf{p H}$ & $21,7.41$ & $23.3,7.455$ \\
BP(mm Hg),HR(/min) & $120 / 70 \mathrm{~mm}, 90$ & $110 / 70,80$ \\
SCORTEN & 0 & 0 \\
\hline
\end{tabular}

(Hb- Hemoglobin, TLC- Total leucocyte count, PC- Platelet count, DLC- Differential leucocyte count, SGOT- Serum glutamate oxalate transaminase, SGPT- Serum glutamate pyruvate transaminase, RBS- Random blood sugar, ALP- Alkaline phosphatase, TB- Total bilirubin, DB- Direct bilirubin, TP- Total protein, ALB- Albumin, BP- Blood Pressure, HR- Heart rate)

believed to initiate this type IV hypersensitivity reaction. Cytotoxic molecules-FasL and granulysin are thought to be responsible for thedisseminated keratinocyte apoptosis in SJS/TEN. ${ }^{1}$ Infact the HLAB ${ }^{\star} 1502$ allele has been linked with phenytoinand carbamazepine induced SJS in Thai population. ${ }^{8}$ Lack of such findings in Caucasians along with the findings of recent studies showing the roles of alleles other than $\mathrm{HLAB}^{\star} 1502$ even in the Han Chinese have exempted the allele from becoming the universal marker. ${ }^{9}$ Additionally. Phenytoin being a strong inducer of CYP450, is linked to induction of oxidative stress and generation of reactive oxygen species. ${ }^{10}$ This may be an additive pathogenetic mechanism.

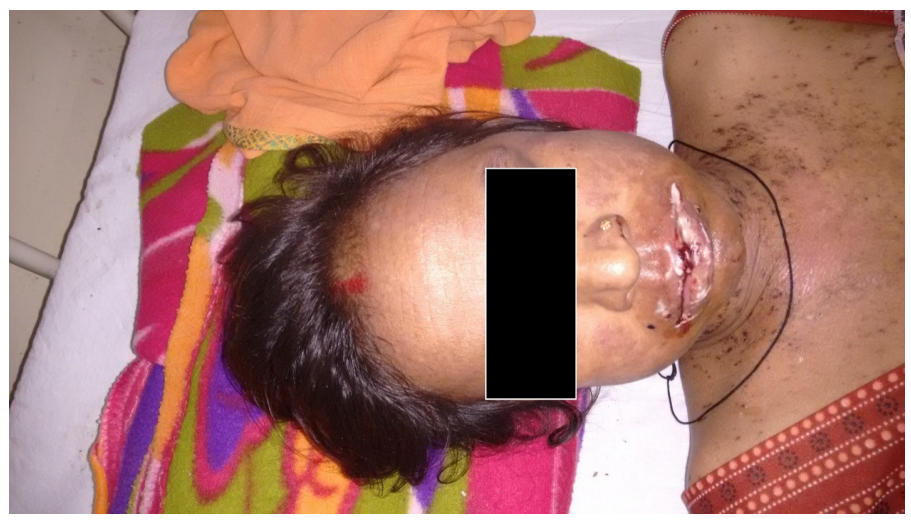

Figure 2: Oral erosions and haemorrhagic crusting

The case highlights the importance of adopting a vigilant approach while prescribing antiepileptics to patients, particularly female seven when the drug is being given for a prophylactic purpose. Regular monitoring of such ADRs, educating physicians and patients can help in early diagnosis and prevent the development of serious consequences of this idiosyncratic reaction.

\section{REFERENCES}

1. Harr T, French LE.Toxic epidermal necrolysis and Stevens-Johnson syndrome. Orphanet J Rare Dis. 2010; 5: 39.

2. Lissia M, Mulas P, Bulla A, RubinoC. Toxic epidermal necrolysis (Lyell's disease). Burns.2009; 36: 152-63

3. Al-QulitiK, Ratrout B, Al-Zaki A. Antiepileptic drugs toxicity: A case of toxic epidermal necrolysis in patient with phenytoin prophylaxis post-cranial radiation for brain metastases.Saudi Pharm J. 2014; 22: 381-4.

4. Bastuji-Garin S, Fouchard N, Bertocchi M, Roujeau JC, Revuz J, Wolkenstein P. SCORTEN: a severity-of illness score for toxic epidermal necrolysis. J InvestDermatol. 2000; 115: 149-53.

5. Patel PP, Gandhi AM,Desai CK, Desai MK, Dikshit RK. An analysis of drug induced Stevens-Johnson syndrome. Indian J Med Res. 2012; 136: 1051-3.

6. Patel TK, Thakkar SH, Sharma DC. Cutaneous adverse drug reactions in Indian population: A systematic review.Indian Dermatol Online J. 2014; 5(Suppl 2): S76-86.

7. Korman NJ, Eyre RW, Zone J, Stanley JR. Drug-Induced Pemphigus: Autoantibodies Directed Against the Pemphigus Antigen Complexes Are Present in Penicillamine and Captopril-Induced Pemphigus. J Invest Dermatol.1991; 96: 273-6.

8. Locharernkul C, Loplumlert J, Limotai C, Korkij W, Desudchit T, Tongkobpetch $\mathrm{S}$, et al. Carbamazepine and phenytoin induced Stevens-Johnson syndrome is associated with HLA-B*1502 allele in Thai population. Epilepsia. 2008; 49: 2087-91.

9. Hua FY, Wua XT, An DM, Yan B, Stefan H, Zhou D. Phenytoin-induced Stevens-Johnson syndrome with negative HLA-B*1502allele in mainland China: Two cases. Seizure. 2011; 20: 431-2.

10. Kandil AO, Dvorak T, Mignano J, Wu JK, Zhu JJ. Multifocal Stevens-Johnson syndrome after concurrent phenytoin and cranial and thoracic radiation treatment, a case report. RadiatOncol. 2010; 5: 49 